\title{
Tex19 and Sectm1 concordant molecular phylogenies support co-evolution of both eutherian-specific genes
}

\author{
Laurent Bianchetti ${ }^{*}$, Yara Tarabay ${ }^{2,5}$, Odile Lecompte ${ }^{3}$, Roland Stote ${ }^{1}$, Olivier Poch ${ }^{3}$, Annick Dejaegere \\ and Stéphane Viville ${ }^{2,4}$
}

\begin{abstract}
Background: Transposable elements (TE) have attracted much attention since they shape the genome and contribute to species evolution. Organisms have evolved mechanisms to control TE activity. Testis expressed 19 (Tex19) represses TE expression in mouse testis and placenta. In the human and mouse genomes, Tex19 and Secreted and transmembrane 1 (Sectm1) are neighbors but are not homologs. Sectm1 is involved in immunity and its molecular phylogeny is unknown.
\end{abstract}

Methods: Using multiple alignments of complete protein sequences (MACS), we inferred Tex19 and Sectm1 molecular phylogenies. Protein conserved regions were identified and folds were predicted. Finally, expression patterns were studied across tissues and species using RNA-seq public data and RT-PCR.

Results: We present 2 high quality alignments of 58 Tex 19 and 58 Sectm 1 protein sequences from 48 organisms. First, both genes are eutherian-specific, i.e., exclusively present in mammals except monotremes (platypus) and marsupials. Second, Tex19 and Sectm1 have both duplicated in Sciurognathi and Bovidae while they have remained as single copy genes in all further placental mammals. Phylogenetic concordance between both genes was significant ( $p$-value $<0.05$ ) and supported co-evolution and functional relationship. At the protein level, Tex 19 exhibits 3 conserved regions and 4 invariant cysteines. In particular, a CXXC motif is present in the N-terminal conserved region. Sectm1 exhibits 2 invariant cysteines and an Ig-like domain. Strikingly, Tex19 C-terminal conserved region was lost in Haplorrhini primates while a Sectm1 C-terminal extra domain was acquired. Finally, we have determined that Tex19 and Sectm1 expression levels anti-correlate across the testis of several primates $(\rho=-0.72)$ which supports anti-regulation.

Conclusions: Tex 19 and Sectm 1 co-evolution and anti-regulated expressions support a strong functional relationship between both genes. Since Tex19 operates a control on TE and Sectm1 plays a role in immunity, Tex19 might suppress an immune response directed against cells that show TE activity in eutherian reproductive tissues.

Keywords: Co-evolution, Tex19, Sectm1, Molecular phylogeny, Expression, CXXC, Mammals, Transposon, Immunity

\section{Background}

Genome stability is required to pass genetic information through generations. However, genetic variations are also crucial to generate organisms that could survive environmental changes. Transposable elements (TE) play a major role in genome evolution [1]. TE act as sites

\footnotetext{
* Correspondence: laurent.bianchetti@igbmc.fr

Annick Dejaegere and Stéphane Viville are co-last authors.

'Biocomputing and Molecular Modelling Laboratory, Integrated Structural Biology Department, Genetics institute of Molecular and Cellular Biology (IGBMC), INSERM U964/CNRS UMR 1704/Strasbourg University, 1 rue Laurent Fries, 67404 Illkirch, France

Full list of author information is available at the end of the article
}

of recombination and can modify the expression of neighbor genes. Among TE, retrotransposons use a RNA intermediate to replicate and integrate back into the genome. Retrotransposons are mainly divided in 3 groups [2]: long interspersed nuclear element (LINE), short interspersed nuclear element (SINE), and long terminal repeat (LTR) also referred to as endogenous retroviruses (ERV). LINE-1, i.e., a sub-class of LINE, encodes two proteins, i.e., ORF1 and ORF2, while ERV encodes gag, pro, pol and env proteins. Organisms have evolved mechanisms to control retrotransposon activity [3], e.g., transcriptional silencing by DNA methylation. 
Recently, the Tex19 gene was proposed to operate a genome defense mechanism against MMERVK10C and LINE-1 retrotransposons in mouse testis and placenta, respectively [4-6]. However, Tex19 molecular mechanism to control TE activity is still unknown.

Tex19 is a mammalian-specific [7] and orphan gene, i.e., the encoded protein does not share sequence similarity with any known proteins [8]. Tex19 shows 2 paralogs in mouse, Tex19.1 and Tex19.2, that code for proteins of 351 and 317 residues, respectively. Initially, human was thought to exhibit no Tex19 ortholog $[9,10]$. However, a human Tex19 gene was detected in GRCh37/ hg19 genome assembly and was predicted to code for a 164 residue protein [7]. Human Tex19 protein is significantly shorter than mouse orthologs because a stop codon prematurely disrupts the coding sequence (CDS). Human Tex19 and mouse Tex19.1 protein sequences share $47 \%$ identity and $56.8 \%$ similarity. In mouse, Tex19.1 and Tex19.2 paralogs share $61 \%$ identity and $69 \%$ similarity and are specifically expressed in reproductive tissues [11]. Briefly, both Tex19.1 and Tex19.2 are expressed in testis while only Tex19.1 is expressed in placenta. Tex19.1 deletion leads to spermatogenesis defects and reduces perinatal survival [6]. Moreover, surviving adult Tex19.1 -/- male mice exhibit activation of MMERVK10C endogenous retroviruses and genomic DNA double strand breaks during meiosis $[4,6]$. Finally, immunohistochemistry experiments localized Tex19.1 protein in the cytoplasm. Tex19.2 role is unknown. In the human and mouse genomes, Tex19 and Sectm1 are neighbors on chromosome cytoband 17q25.3 and 11qE2, respectively, but are not homologs.

A Sectm1 cDNA was originally cloned from human K562 erythroleukemic cells and codes for a 248 aminoacid protein which exists as a Golgi localized type I transmembrane (TM) protein and a secreted isoform [12]. Mouse exhibits 2 Sectm 1 paralogs, i.e., Sectm1a and Sectm1b, that are both expressed in a large variety of unrelated tissues $[13,14]$, e.g., colon, kidney, stomach, testis, placenta, lung, thymus, spleen and liver. So far, Sectm1 has exclusively been studied in human and mouse and its phylogeny is unknown. Interestingly, Sectm1 binds CD7 [13], an Ig domain protein which is encoded by Sectm1 neighbor gene in the human and mouse genomes. In both organisms, Tex19, Sectm1 and CD7 are located in syntenic chromosome regions. In mouse, gene knock-out (KO) studies of Sectm1 or CD7 have not been carried out. CD7 is expressed on T, NK and pre-B lymphocytes. Sectm1 and CD7 protein complex modulates T-cell activation. In addition, Sectm1 expression is stimulated by interferon gamma (IFN-gamma) [15]. Sectm1 is thus clearly involved in the immune system.

Comparative genomics holds tremendous promises to fill the gap between sequence, structure, function and evolution. With the multiplication of $2^{\text {nd }}$ and $3^{\mathrm{d}}$ generation sequencers [16, 17], large genomes can now be sequenced at an unprecedented pace and more than 50 mammalian genome projects are under way [18]. Mammals form a complex group of approximately 5,300 species divided in Prototheria (monotremes), Metatheria (marsupials) and Eutheria (usually called placental mammals). Eutheria are further divided in 4 groups, i.e., Xenarthra (e.g., armadillo and sloth), Afrotheria (e.g., elephant and rock hyrax), Laurasiatheria (e.g., horse and shrew) and Euarchontoglires (e.g., human and mouse) [19]. Species have been thoroughly selected for whole genome sequencing to achieve divergence across mammals and enable biologists to carry out unbiased molecular phylogenies. Classically, biologists wish to study gene evolutionary behavior by analyzing presence/ absence, copy number, evolutionary rates, and genetic loci reshuffling across genomes. Such research projects may also benefit from expression data to provide support to biological conclusions. Complete genomes are particularly valuable because they provide quite definitive answers to gene presence/absence studies [20]. However, "finished" genome sequences may still contain some gaps. So far, only a few mammalian genomes have been completed, namely Ornithorhynchus anatinus (platypus) (Prototheria) [21], Monodelphis domestica and Macropus eugenii (Methateria) [22], and human and mouse (Eutheria). In a genome project, sequence annotation is a crucial step. Bioinformatics pipelines annotate sequences on a genome wide scale and pave the way to gene evolution studies. In particular, multiple alignments of complete protein sequences (MACS) [23] have proved invaluable to investigate sequence, structure, function and evolution relationships for countless proteins. Recently, specialized bioinformatics tools have made it possible to detect co-evolving genes [24]. Co-evolving proteins are comparative genomics niceties. Such proteins are characterized by concordant molecular phylogenies, i.e., shared history of gene duplication and gene losses, and similar patterns of presence and absence across species [25]. In addition, co-evolution tends to indicate functional relationship, physical interaction or same biological process participation. Co-evolution has been reported for hormones and receptors, e.g., insulin [26] and vasopressin [27], protease and substrate, e.g., hatching enzyme and egg envelope protein [28]. Cis proximity of co-evolving genes along the chromosome is not required, e.g., the insulin and its receptor are coded by genes located within human chromosome 11 and 19 [26], respectively. In prokaryotes, gene order along the genome and function are related and genes are organized in operons [29]. By contrast, operons are very rare in eukaryotes [30, 31]. Only a few gene systems, e.g., Hox, $\beta$-globin, GPAT/AIRC [32] and invertebrate species [33] exceptions have been reported. As a result, cis 
proximity of genes along eukaryote chromosomes does not relate to function and molecular phylogeny.

High-throughput (HTP) transcript sequencing across tissues and species now enables biologists to study gene expression through the lens of evolution [34, 35]. However, molecular phylogenetic analysis is recommended before profiling gene expression [36, 37]. Gene expression patterns across tissues and species may provide valuable information to elucidate gene function. Among expression profiling methods, RNA-seq has been a breakthrough to quantify gene expressions on a genome wide scale. A plethora of RNA-seq experiments carried out in mammalian tissues has been made publicly available in the sequence read archive (SRA) database [38].

Using a 13 protein sequence alignment, we previously established the mammalian specificity of Tex19 [7]. The recent availability of a series of mammalian genome sequences for Prototheria, Metatheria and Eutheria prompted us to revisit Tex19 molecular phylogeny. Intriguingly, we noticed that both Tex19 and Sectm1 were unique in human but duplicated in mouse and rat, i.e., a quite unusual gene copy number pattern. In addition, both genes were neighbors on human, mouse and rat genomes. This unusual gene system and loci proximity in human and rodents encouraged us to simultaneously investigate Tex19 and Sectm1 molecular phylogenies. In the present study, 2 sequence homology searches have been carried out in protein databases and 2 high quality MACS of 58 proteins have been built. First, the sequence homologs searches show that both genes are eutherian-specific. Second, Tex19 and Sectm1 have duplicated in Sciurognathi and Bovidae while they have been maintained as single copy genes in all further placental mammals. The remarkable concordance of the evolutionary histories of the two genes, and in particular the duplications in the same subset of species strongly suggests co-evolution and functional relationship. Finally, Tex19 and Sectm1 expressions were analyzed across tissues and species and enabled us to detect pattern oppositions, e.g., human placenta expresses Sectm1 but not Tex19 while it is exactly the opposite in mouse placenta. Since Tex19 represses TE expression in reproductive tissues and Sectm1 plays a role in immunity, our results suggest a new eutherian-specific mechanism that might prevent the immune system from attacking tissues where controlled TE activity occurs.

\section{Methods}

\section{MACS construction}

We used PipeAlign [39] to generate the MACS. Homologs of mouse full-length Tex19.1 and human full-length Sectm1 proteins were searched in Uniprot, RefSeq and the Protein Data Bank (PDB) using BLASTp (expect threshold $10^{-4}$, word size 3, BLOSUM62, gop 11 and gep 1). For
Tex19 and Sectm1 homolog searches, the score, identities and positives cut-off values were set to 65 bits, $28 \%$ and $40 \%$, respectively. In addition, whole genome shotguns (WGS) were searched by tBLASTn for further homologs. The mouse Tex19.1 sequence was used as a query because human Tex19 lacks the C-terminal region. Conversely, the human Sectm1 sequence was used as a query because mouse Sectm1 proteins lack the C-terminal region. Alignment quality was manually refined in the SeqLab (GCG, Wisconsin package) editor by moving residues. For each protein, basic data, i.e., accession number, organism, brief taxonomy, sequence length and coding origin were reported in a file (publicly available on the FigShare repository http://figshare.com/articles/Catalog_of_Tex19_ protein_sequences/1491365 and http://figshare.com/ articles/Catalog_of_Sectm1_protein_sequences/1491366). Multiple alignment schemas were drawn in Jalview 2 [40]. Sequence logos were created in WebLogo 2.8.2 [41].

\section{Sequence comparisons and secondary structure predictions}

Pairwise sequence comparisons were carried out with Needleman \& Wunsch or Smith \& Waterman algorithms implemented in the needle and water EMBOSS tools (Olson SA) (default parameters), respectively. Secondary structures were predicted with PSIPRED (Jones DT). Position-specific-iterated BLAST (PSI-BLAST) were carried out in the NCBI nr protein database with default parameters to search for remote homologs of Tex19 and Sectm1. Ten and three iterations were carried out for Tex19 and Sectm1, respectively.

\section{Molecular modelling}

A HHpred search was carried out in the PDB with human Sectm1 as a query. The [PDB:3SOB] structure (X-ray, 1.9 Angström resolution) was selected as a template to model by homology Sectm1 Ig-like domain in the Modeller 9.13 program (Sali A). Sectm1 3D model was validated using MolProbity (Chen V. B. et al., 2010) and PROSA (Wiederstein M. and Sippl M. J., 2007). 3D structures were analyzed in PyMOL 1.4.1 (Schrödinger, LLC.).

\section{Molecular phylogeny}

The maximum likelihood (ML) algorithm implemented in MEGA6 [42] was applied to build the phylograms of all full-length sequences. Using ProtTest [43], the JonesTaylor-Thornton (JTT) residue substitution model was selected as the most appropriate. A 500 replicate bootstrap was carried out. Finally, phylograms were annotated with the iTOL software [44].

\section{RNA-seq profiles across tissues and species}

RNA-seq experiments were selected from the SRA database. Tex19 and Sectm1 transcripts were masked for 
interspersed repetitive elements (IRE) (RepeatMasker, Smit A, unpublished) and used as queries on selected RNA-seq experiments. Reads were mapped to Tex19 and Sectm1 masked transcripts using MegaBLAST. Thanks to its default large word size $(\mathrm{W}=28)$, MegaBLAST selectively retrieves sequences that are highly similar to a query. In double paralog species, i.e., mouse, rat and cow, a best score approach was applied to reliably map reads to their proper paralog. Ex-aequo scoring reads were discarded. Homo sapiens [RefSeq:NM_207459], Pan troglodytes [RefSeq:NM_001246469], Pan paniscus [RefSeq:XM_ 003808957], Papio anubis [RefSeq:XM_003913613] and Macaca mulatta [RefSeq:NM_001194590] Tex19 transcripts were used as queries. Sectm1 expression was searched with Homo sapiens [RefSeq:NM_003004], Pan troglodytes [RefSeq:XM_001167936], Papio anubis [RefSeq:XM_003913612] and Macaca mulatta [Genbank:EHH25327] transcript sequences as queries. Mouse experiments were searched with Tex19.1 [RefSeq:NM_028602], Tex19.2 [RefSeq:NM_027622], Sectm1a [RefSeq:NM_145373] and Sectm1b [RefSeq: NM_026907]. Finally, cow Tex19.alpha [RefSeq:XM_ 002696130], Tex19.beta [RefSeq:XM_001251766], Sectm1. alpha [RefSeq:NM_001102326] and Sectm1.beta [RefSeq: NM_001083787] were used as queries on cow RNA-seq experiments.

\section{EST profiles}

Human and cow placenta ESTs were automatically selected from Genbank release 199 using taxonomy and tissue_type fields. Genbank records were converted to BLAST databases. Human Sectm1 [RefSeq:NM_003004] and cow Tex19.alpha [RefSeq:XM_002696130], Tex19.beta [RefSeq:XM_001251766], Sectm1.alpha [RefSeq:NM_ 001102326] and Sectm1.beta [RefSeq:NM_001102326] masked transcripts were used as queries. MegaBLAST was used to search the EST database. For cow, ESTs were properly mapped to Tex19 or Sectm1 paralogs using a best score approach. Ex-aequo scoring ESTs were discarded.

\section{Reverse-transcription polymerase chain reaction (RT-PCR)} RNA from selected tissues was prepared using the MACHEREY-NAGEL protocol of the NucleoSpin RNA L kit following the manufacturer's instructions. After DNase I digestion (Roche), $2 \mu \mathrm{g}$ RNA was reverse-transcribed by random priming using Superscript II (Invitrogen). Resulting cDNA was diluted in a final volume of $80 \mathrm{ml} .1 \mu \mathrm{L}$ of each cDNA was used to perform 40 cycles PCR amplification of $94{ }^{\circ} \mathrm{C}$ for $30 \mathrm{~s}, 64{ }^{\circ} \mathrm{C}$ for $1 \mathrm{~min} 30 \mathrm{~s}$ and $72{ }^{\circ} \mathrm{C}$ for $1 \mathrm{~min}$ 30s. PCR products were checked by sequencing (GATC) to validate the specificity of the primers. In rat, 5' -ATGTGTCCCCCAGTCAGTGTT-3' (forward) and 5' TCAAGGGAAGAAGGATCGAGCA-3' (reverse) primers were used to target Tex19.1 (NM_001017482) while 5' -ATGTGTCCCCCAGTCAGTGTT-3' (forward) and 5' TTAGTTGTGTGGCTCAGGGGA-3' (reverse) targeted Tex19.2 (NM_001109622). In cow, 5'-CGATTCTGAGG CATGGTCTG-3' (forward) and 5' GTATGATTGTG CAAGCCCAC 3' (reverse) primers were designed to target the XM_002696130 transcript sequence, which codes for XP_002696176, i.e., Bos taurus Tex19.alpha. 5'-AACTCA GAGACAGGGTCTGA-3' (forward) and 5'-AAGTGAT CATCTAGGTCCAT-3' (reverse) primers targeted the XM_005195806 transcript which codes for XP_005195863, i.e., Bos taurus Tex19.beta. 5'-ATGGTGAAGGTCGG AGTGAA-3' (forward) and 5'-TTACTCCTTGGAGGC CATGT-3' (reverse) primers were used to target the cow GAPDH transcript.

\section{Results}

\section{Tex19 and Sectm1 are not homologs}

A dot plot was drawn to determine if Tex19 and Sectm1 proteins exhibit sequence similarity (Additional file 1). As controls of sequence homology, human Tex19 and Sectm1 were compared to mouse Tex19.1 and Sectm1a orthologs, respectively. Human Tex19 and Sectm1 proteins do not show any similarity while homology between human and mouse orthologs was obvious. To determine if Tex19 and Sectm1 proteins might be distant homologs, i.e., structure was conserved while sequences diverged, folds were investigated. First, Sectm1 structure is unknown. However, a weak sequence similarity with the Ig domain was previously mentioned [12]. Second, Tex19 structure has not been resolved yet. Using Tex19 protein sequence as a query, a conserved domain database (CDD) search did not produce any hit. Therefore, Tex19 unlikely folds into an Ig domain. In conclusion, both proteins do not share sequence homology and are unlikely distant homologs.

\section{Tex19 and Sectm1 neighbor genes are unique in human and duplicated in mouse and rat}

In human, the presence of a Tex19 ortholog has been controversial $[7,9]$. Therefore, we wished to determine the exact number of homologs on the latest human genome assembly. In our hands, exhaustive sequence similarity searches on the reference GRCh37.p13/hg19 genome showed that tex19, but also sectm1 are unique. Moreover tex19 and sectm1 are neighbors (Fig. 1) and localize on cytoband 17q25.3 on the human genome. A Tex19.1 and Tex19.2 paralogy was previously reported in mouse and rat [7]. Tex19.1 and Tex19.2 genes both localize on mouse chromosome cytoband 11qE2. To determine if the mouse genome codes for further Tex19 homologs, an exhaustive tBLASTn search with Tex19.1 as a query was carried out. Two significant hits were produced, both on chromosome 11. Therefore, Tex19 


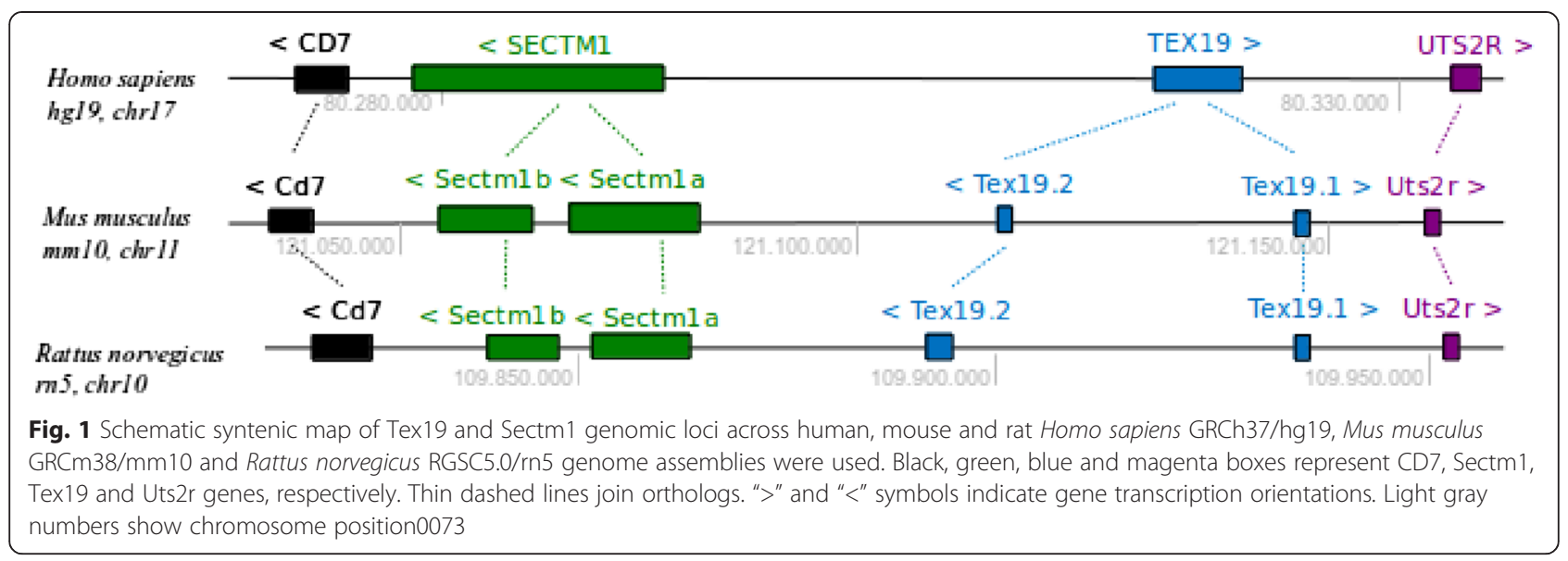

shows 2 paralogs in mouse. An exhaustive search on the mouse genome with Sectm1a as a query produced 2 significant hits, both on chromosome 11. As a result, mouse exhibits also 2 Sectm 1 paralogs. In rat, Tex19.1 and Tex19.2 genes are both localized on chromosome cytoband 10q32.3. Using the same approach, we determined the presence of 2 Tex19 and 2 Sectm 1 genes in rat. Conclusively, Tex19 and Sectm1 are unique in human while duplicated on mouse and rat genomes. We compared Tex19 and Sectm1 genomic loci across species. Tex19 and Sectm1 are neighbors on human, mouse and rat chromosome cytoband 17q25.3, 11qE2 and 10q32.3, respectively. Since the rodents and human radiation, i.e., 90 million years ago, Tex19 and Sectm1 gene copies have been evolutionarily kept close to each other on the genome. In human, tex19 and sectm1 are separated by a $25 \mathrm{~kb}$ intergenic region and both genes are transcribed in opposite directions. In mouse and rat, Tex19.2 and Sectm1a are separated by 35 and $31 \mathrm{~kb}$ DNA regions, respectively. In the 3 species, CD7 is $5^{\prime}$ to Sectm 1 while the Urotensin 2 receptor (Uts2r) is $3^{\prime}$ to Tex19. The chromosome region between CD7 and Uts2r spans approximately 63, 126, and $149 \mathrm{~kb}$ in human, mouse and rat, respectively. While no functional relation has been reported between Tex19 and Uts2r, Sectm1 protein binds CD7. Sectm1 and CD7 are thus clustered genes on the genome. In both rodents, Sectm1a and Sectm1b are transcribed in the same direction while Tex19.1 and Tex19.2 show opposite transcription directions.

\section{Tex19 and Sectm1 are both eutherian-specific}

Using sequence homology searches across genomes and an alignment of 13 Tex 19 proteins originating from 10 species, we previously reported Tex19 mammalian specificity [7]. Now, we present an extended alignment of 58 proteins from 48 organisms supporting Tex19 eutherian specificity (available at http://figshare.com/
articles/Tex19_multiple_alignment_of_complete_protein_ sequences/1491363). Exhaustive tBLASTn searches across prokaryote, plant, yeast, invertebrate, fish, and bird genomes retrieved no Tex19 homologs (Table 1). Moreover, Ornithorhynchus anatinus (platypus), Macropus eugenii (wallaby) and Monodelphis domestica (opossum) complete genomes did not produce any significant hit. Therefore, Tex19 is absent in monotremes and marsupials. Tex19 was further searched in the genomes of organisms that represent the 4 super-orders of eutherians (Additional file 2), i.e., Afrotheria, Laurasiatheria, Euarchontoglires and Xenarthra. Without ambiguity, Tex19 homologs were detected in Afrotheria (3 sequences from 3 species), Laurasiatheria (25 sequences from 20 species) and Euarchontoglires (30 sequences from 25 species). No Tex19 homolog was detected in Xenarthra. Since Xenarthra genomes, e.g., Dasypus novemcinctus (armadillo) and Choloepus hoffmanni (sloth) are not finished yet, we cannot conclude that Tex19 does not exist in those species. Thus, except in Xenarthra where Tex19 presence/absence is ambiguous, Tex19 appears to be eutherian-specific. A pairwise sequence comparison of human and mouse Sectm1 proteins was previously published [14]. We present a Sectm1 MACS of 58 proteins from 48 organisms (available at http://figshare.com/articles/Sectm1_multiple_alignment_of_complete_protein_sequences/1491364). First, Sectm1 is absent in non-mammalian species, monotremes and marsupials (Table 1). Second, Sectm1 homologs have been detected in Afrotheria ( 5 sequences from 5 species), Laurasiatheria (28 sequences from 23 species), Euarchontoglires (24 sequences from 19 species) and Xenarthra (1 sequence from 1 species). Indeed, the Dasypus novemcinctus [RefSeq:XP_004478808] protein shares significant sequence similarity with the human Sectm 1 ( $45.7 \%$ identity and $56.3 \%$ similarity). Therefore, we can conclude that Sectm1 is present in the genome of organisms that represent the 4 super-orders of eutherians. Thus, Sectm1 also appears to be eutherian-specific. 
Table 1 Tex19 and Sectm1 presence/absence across species

\begin{tabular}{|c|c|c|c|c|}
\hline \multicolumn{5}{|c|}{ Eutherian specificity of Tex19 and Sectm1 } \\
\hline Tex19 & Sectm1 & Organism & Clade & Eutheria \\
\hline A & A & Escherichia coli ${ }^{\mathrm{a}}$ & Bacteria & no \\
\hline A & A & Saccharomyces cerevisiae ${ }^{a}$ & Fungi & no \\
\hline A & A & Arabidopsis thaliana $^{a}$ & Plant & no \\
\hline A & A & Caenhorhabditis elegans ${ }^{a}$, Drosophila melanogaster & Invertebrate & no \\
\hline A & A & Brachydanio rerio $^{a}$ & Fish & no \\
\hline A & A & Gallus gallus & Bird & no \\
\hline A & A & Ornithorhynchus anatinus ${ }^{a}$ & Monotreme & no \\
\hline A & A & Macropus eugenii $i^{\mathrm{a}}$, Monodelphis domestica ${ }^{\mathrm{a}}$ & Marsupial & no \\
\hline A & $P$ & Dasypus novemcinctus ${ }^{\mathrm{b}}$ & Xenarthra & yes \\
\hline P & P & Bos taurus & Bovidae & yes \\
\hline P & P & Cavia porcellus & Hystricognathi & yes \\
\hline P & P & Mus musculus ${ }^{\mathrm{a}}$, Rattus norvegicus & Sciurognathi & yes \\
\hline$P$ & $P$ & Homo sapiens ${ }^{a}$, Pan troglodytes ${ }^{a}$, Macaca mulatta & Primate & yes \\
\hline
\end{tabular}

$A$ absent, $P$ present, ${ }^{(a)}$ complete genome, ${ }^{(b)}$ requires finished genome confirmation

\section{Remarkable concordance of Tex19 and Sectm1 molecular phylogenies}

Tex19 and Sectm1 concordant gene copy numbers in human, mouse and rat prompted us to infer the molecular phylogenies of both genes across species. First both genes are eutherian-specific. Second, both genes duplicated in Sciurognathi (a rodent suborder) and Bovidae (a family of Ruminantia) (Fig. 2). Third, in all further Eutheria, Tex19 (Fig. 2a) and Sectm1 (Fig. 2b) were both present as single-copy genes. Of note, Sciurognathi and Bovidae orthologs do not cluster together in the phylograms which supports 2 independent gene duplication events. We used 3 methods to test statistically the concordance of Tex19 and Sectm1 phylogenetic histories across Eutheria. First, we applied an independence Pearson's Chi-squared test. Tex19 and Sectm1 gene copies were counted across 8 selected eutherian species from Xenarthra to human (Table 2) and a contingency

Table 2 Tex19 and Sectm1 gene copies across species

\begin{tabular}{|c|c|c|c|}
\hline \multicolumn{4}{|c|}{ Gene copies of Tex19 and Sectm1 across Eutheria } \\
\hline Tex19 & Sectm 1 & Eutheria & Clade \\
\hline$\overline{0}$ & 1 & Dasypus novemcinctus ${ }^{b}$ & Xenarthra \\
\hline 1 & 1 & Homo sapiens ${ }^{a}$ & Primate \\
\hline 1 & 1 & Pan troglodytes ${ }^{a}$ & Primate \\
\hline 1 & 1 & Macaca mulatta & Primate \\
\hline 1 & 1 & Cavia porcellus & Hystricognathi \\
\hline 2 & 2 & Mus musculus ${ }^{a}$ & Sciurognathi \\
\hline 2 & 2 & Rattus norvegicus & Sciurognathi \\
\hline 2 & 2 & Bos taurus & Bovidae \\
\hline
\end{tabular}

table was drawn (Table 3). A $0.018 p$-value was calculated and was below $5 \%$ type I error risk. Therefore, we concluded that Tex19 and Sectm1 gene copy numbers were not independent across Eutheria which supports co-evolution. Second, identity percent vectors to a selected reference sequence, i.e., human, were used to calculate the correlation between both protein sequences across Eutheria [25]. Pairwise identity percents were calculated for each protein in the MACS. Two identity percent vectors were generated, 1 for Tex19 and 1 for Sectm1, and correlated at 0.78 (Pearson) providing further support to Tex19 and Sectm1 co-evolution. Third, the MirrorTree server (Ochoa D. and Pazos F., 2010) was used to calculate the similarity between both trees (all sequences). 39 species were common to both phylograms, a 0.717 correlation and a $p$-value $<=10^{-6}$ were calculated and supported co-evolution.

\section{Tex19 shows 4 invariant cysteines and Haplorrhini lost a conserved C-terminal region}

Basing our analysis on a 13 sequence alignment, we previously reported the presence of 3 conserved regions in Tex19 that we named "MCP", "VPTEL" and "non-primate specific" from the $\mathrm{N}$ to the C-terminus. Now, the 58 Tex19 MACS enables us to confirm those 3 conserved regions and the invariance of the Cys-2 and Glu-123 (offsets refer to human Tex19) that are involved in the "MCP" and "VPTEL" motifs (Fig. 3a), respectively. The "nonprimate specific" region is conserved in all eutherian species including Strepsirrhini, e.g., Otolemur garnettii, except in Haplorrhini primates that remarkably lost this region (Fig. 3a). Since Strepsirrhini are primates, the C-terminal region cannot be further called "non-primate 

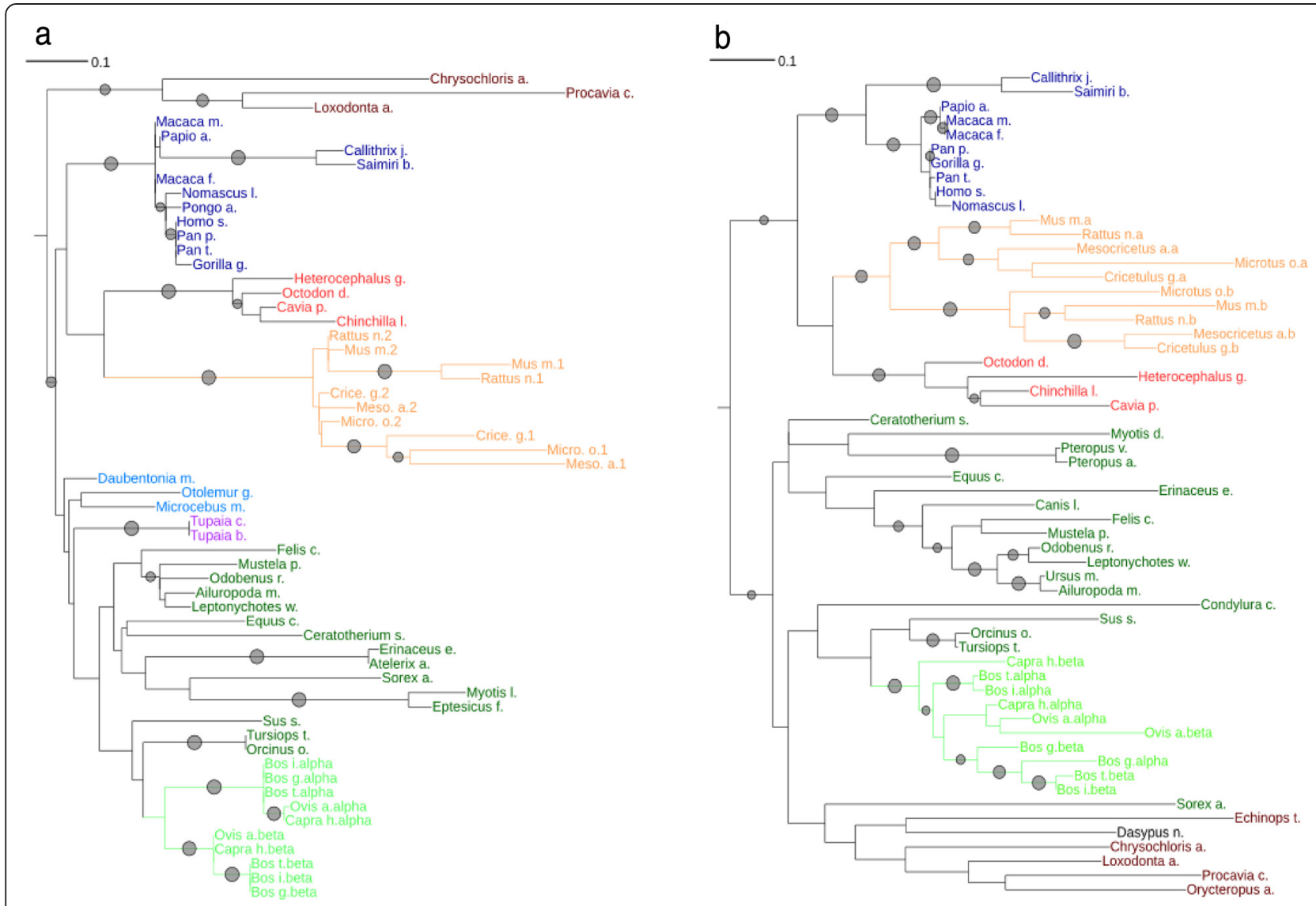

Fig. 2 Tex19 and Sectm1 ML phylograms of all eutherian sequences. a Tex19 and b Sectm1. Bootstrap values greater than $65 \%$ are symbolized by gray circles. Taxonomic groups of species are colored as follows: Haplorrhini = dark blue, Strepsirrhini = light blue, Scandentia = purple, Afrotheria $=$ dark brown, Hystricognathi $=$ dark red, Sciurognathi $=$ orange, Bovidae = light green, further Laurasiatheria = dark green. Orange and light green branches depict Sciurognathi and Bovidae lineage sequences, respectively. Sectm1 sequences of Strepsirrhini species were not available in protein databases likely because genomes were still incomplete

specific" and shall be renamed. Remarkably, the 58 Tex19 MACS reveals 4 invariant cysteines. In the human sequence, 3 invariant cysteines are localized at position 2, 34 and 37, i.e., in the "MCP" region, and the latter at position 155 , i.e., in the "VPTEL" region. Cys-34 and Cys-37 are involved in a $\mathrm{C}_{34} \mathrm{~F}[\mathrm{AT}] \mathrm{C}_{37}[\mathrm{FY}]$ motif (Fig. $3 \mathrm{~b}$ ) which fits the fuzzier CXXC pattern. The "MCP" region contains also a highly conserved histidine at position 27 . Moreover, a further invariant residue, i.e., His-157, is present in the "VPTEL" region. In the previously called "non-primate specific" region, a series of leucine amino-acids are invariant at positions $186,188,240,241,270,273,282$, and 289 (offsets refer to mouse Tex19.1 protein). Therefore,

Table 3 Eutheria contingency table by Tex19 and Sectm1 gene copy pattern

\begin{tabular}{llll}
\hline & Tex19: 0 copy & Tex19: 1 copy & Tex19: 2 copies \\
\hline Sectm1: 1 copy & 1 & 4 & 0 \\
Sectm1: 2 copies & 0 & 0 & 3 \\
\hline
\end{tabular}

we propose to rename the "non-primate specific" region as "iLeu" for invariant leucines. Tex19 does not show any sequence similarity with known proteins or domains. Strategies have been described to detect remote homology [45], i.e., fold conservation between proteins that share weak primary sequence similarity. Using the human "MCP" region sequence (Met-1 to Trp-54) as a query, remote homolog search algorithms, e.g., PSI-BLAST, Interproscan and HMMER, did not produce any significant hit. Human Tex19 "MCP" region was submitted to PSIPRED secondary structure prediction program which predicted 2 alpha-helices (Fig. 3c). The first helix spans residues Ser-15 to His-27 with a $7.4+/-1.8$ confidence value while the second helix spans residues Ser-32 to Ser51 with a $6.05+/-2.8$ confidence value (maximum confidence is 9). Invariant Cys-2 and His-27 amino-acids were predicted in a coil region and in the first alpha-helix, respectively. Invariant $\mathrm{Cys}-34$ and $\mathrm{Cys}-37$, i.e., the $\mathrm{C}_{34} \mathrm{~F}[\mathrm{AT}] \mathrm{C}_{37}[\mathrm{FY}]$ motif, were both predicted in the second alpha-helix. 
a

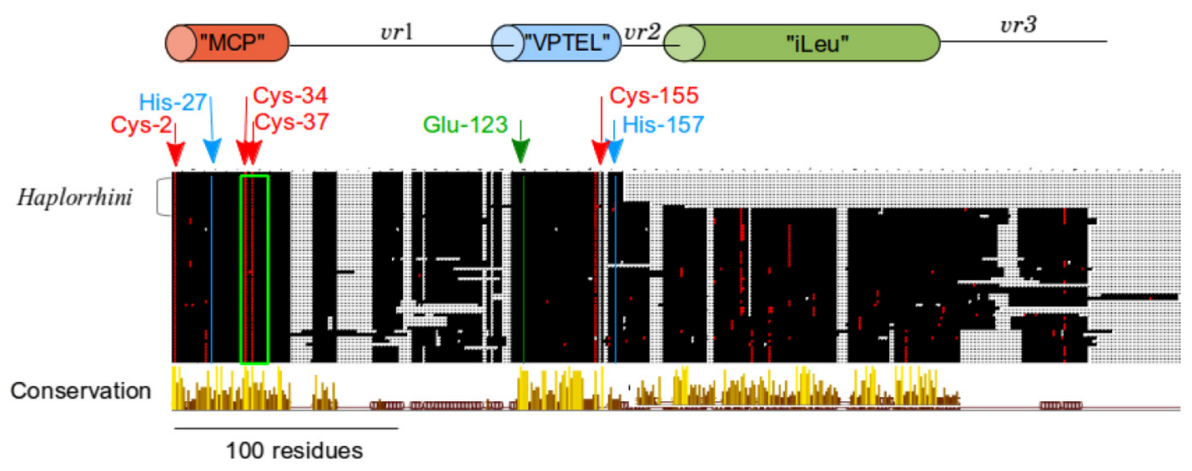

b

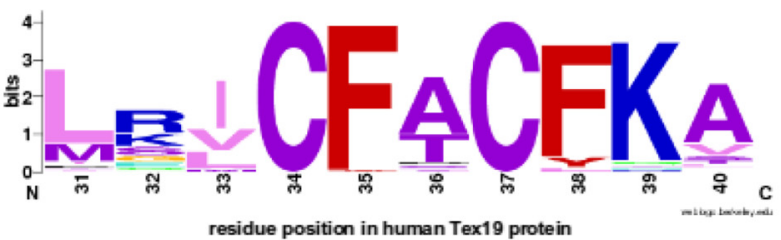

C

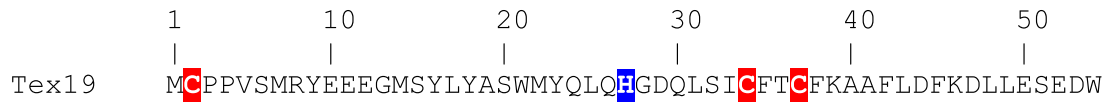

PsiPred CCCCCCCCCCCCCCHнHнHнHнHнHнHCCCCHнHнHнHнHнHнHнHнHнHнCCC

Conf. $\quad 988711224336725788999898763685124677458988988986500799$

Fig. 3 Tex 19 protein organization. a MACS schema. Colored cylinders represent conserved regions while "vr" stand for variable regions. Cysteines are depicted as red dots while any other residues, except invariant amino-acids, are shown in black. Gray surfaces represent gaps. Arrows indicate invariant residues and offsets refer to the human sequence, i.e., [RefSeq:NP_997342]. The open green rectangle shows the position of the $C_{34}\left[\mathrm{~F}[\mathrm{AT}] \mathrm{C}_{37}[\mathrm{FY}]\right.$ motif. Haplorrhini sequences were clustered at the top of the alignment. b $\left.C_{34}[A]\right] C_{37}[F Y]$ logo. c Secondary structure prediction in conserved "MCP" region (PsiPred), i.e., from residue Met-1 to Trp-54. $\mathrm{H}$ and C symbols represent alpha-helix and coil predicted residues, respectively. Confidence value of secondary structure prediction is reported for each residue (maximum confidence equals 9). Invariant cysteines are depicted with a red background while invariant His-27 is shown with a blue background

\section{Sectm 1 exhibits an Ig-like fold with an atypical disulfide bridge and Haplorrhini acquired an extra C-terminal region}

No multiple alignment of Sectm1 has been proposed so far. However, a secretion peptide (SP), a transmembrane (TM), and a weak sequence similarity with the Ig domain were previously mentioned [12]. Using the 58 Sectm1 MACS, we report the presence of a 100 residue conserved region, that we called "A" (Fig. 4a). Strikingly, Haplorrhini primates exhibit an extra C-terminal region that we named $\mathrm{B}$. Conserved region A spans residue Trp-33 to Val-133 (residue offsets refer to the human sequence). Region A shows 2 invariant cysteines, i.e., Cys-38 and Cys-55. In addition, a strongly conserved motif containing 6 invariant residues $\mathrm{G}_{113} \mathrm{XY}_{115} \mathrm{XW}_{117}$ $\mathrm{XL}_{119} \mathrm{XG}_{121} \mathrm{XQ}_{123}$ (Fig. 4b) is present in region $\mathrm{A}$. $\mathrm{A}$ sequence similarity between Sectm1 and the Ig domain was previously reported using BLASTp or FASTA tools [12]. Using PSI-BLAST and only 3 iterations, we were also able to reproduce this result and a significant sequence similarity was found between human Sectm1 conserved region $\mathrm{A}$ and the human $\mathrm{B}$-cell antigen receptor complexassociated protein alpha chain (CD79A, [RefSeq: NP_067612]) (Table 4). A conserved domaine database (CDD) search with human CD79A sequence as a query retrieved the Ig domain without ambiguity (E-value = 3.85e-08). However, CD79A structure has not been resolved yet. Therefore, CD79A could not be used as a template for homology modelling. Using human Sectm1 sequence from residue 1 to 186 (i.e., residues that aligned on CD79A) as a query, a HHpred search [46] in the structure database retrieved [PDB:3SOB_L] (Score 48.5, $\mathrm{E}$-value $=0.0013$ ), i.e., an antibody light chain (Table 5). A predominant feature of most Ig domains is a sandwich of 

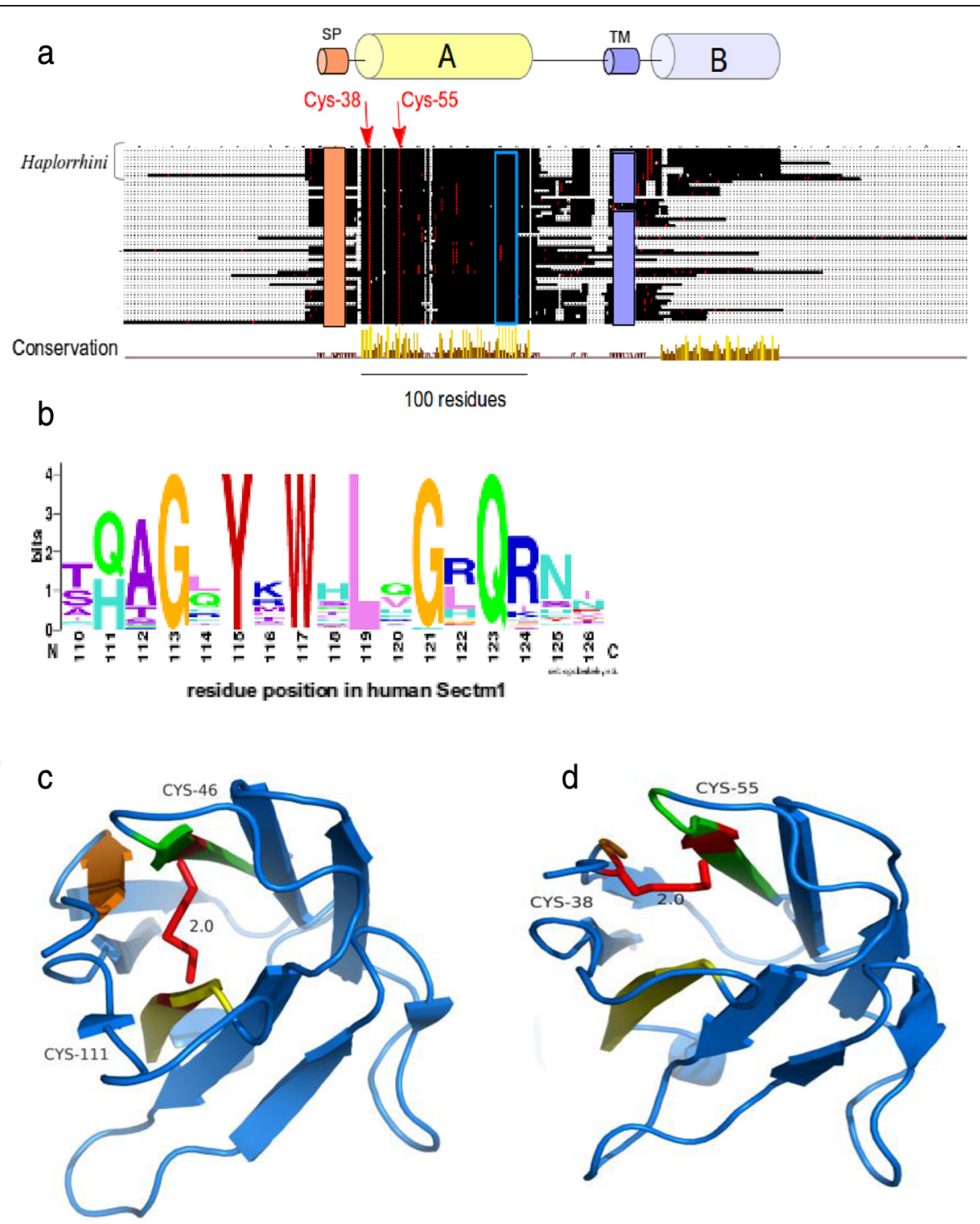

Fig. 4 Sectm1 protein organization. a MACS schema. Colored cylinders represent conserved regions, e.g., region A is the Ig-like domain, while thin lines stand for variable regions. Cysteines are depicted as red dots while any other residues are shown in black. The orange rectangle symbolizes a SP. The violet rectangle delineates a TM. The open blue rectangle shows the position of the $G_{113} X Y X W X L X G X Q_{123}$ highly conserved motif. Arrows indicate 2 invariant cysteines. Amino-acid offsets refer to the human sequence, i.e., [RefSeq:NP_002995]. Haplorrhini sequences were clustered at the top of the alignment. $\mathbf{b}$ Highly conserved $\mathrm{G}_{113} X Y X W X L X \mathrm{CXQ}_{123}$ motif logo; (c) Classical Ig domain [PDB:3SOB] used for homology modelling showing a disulfide bridge (red) between beta-sheets. $\mathbf{d}$ 3D model of Sectm $1 \mathrm{lg}$-like domain, the atypical disulfide bond that is predicted to join both invariant cysteines is depicted in red

2 beta-sheets connected by a disulfide bridge [47]. The classical disulfide bridge was present in [PDB:3SOB_L] template structure between Cys-46 and Cys-111 (Fig. 4c). Using 3SOB_L as a template, the 3D structure of Sectm1 Ig-like domain could be modelled (Fig. 4d). The model was submitted to ProSA [48] which calculated an overall quality score of -4.21 , i.e., in the range of native protein structures. Knowledge-based energy of the molecule was in an acceptable range. Moreover, using MolProbity, Phi and Psi dihedral angles of the Sectm1 Ig-like model were checked. $96 \%$ (96/100) of all residues showed dihedral angles in Ramachandran plot favored regions. However, a peculiarity of the Sectm1 sequence is that the conserved cysteine are rather close in sequence (Cys-38 and Cys-55) and situated in N-terminal of the fold with respect to classical Ig-domains, and they were therefore not aligned with those of the template. To ensure that it is possible to conserve the Ig-domain topology while forming a disulfide bridge between the conserved cysteine, a restraint was applied to bridge both Sectm1 invariant cysteine to 
Table 4 Template selection for homology modelling of Sectm1 Ig-like domain

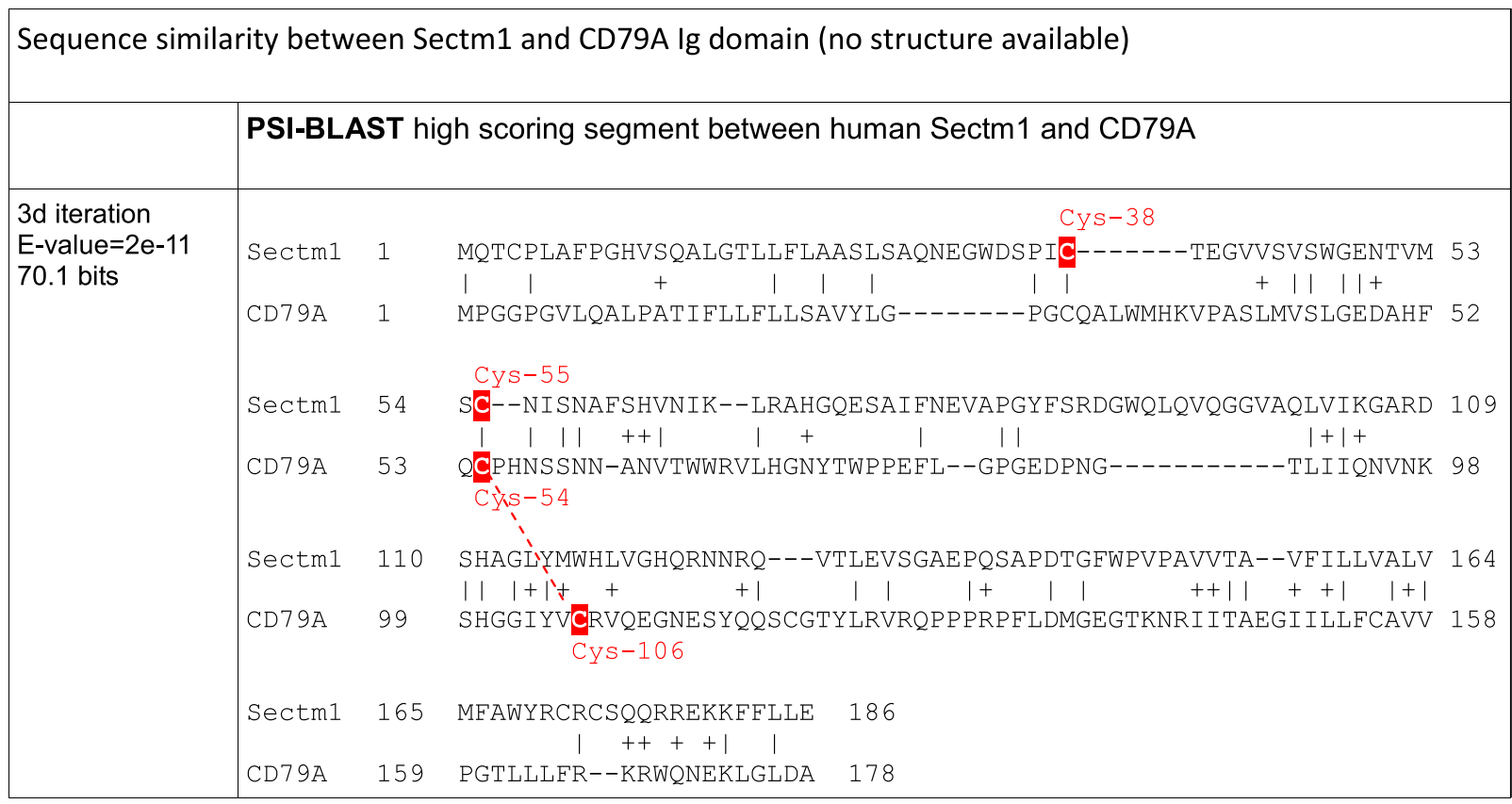

Invariant cysteines are highlighted in red

CD79A putative disulfid bond is depicted as a red dashed line

construct the 3D model. The resulting disulfide bridge did not connect both beta-sheets as the classical Ig disulfide bridge does but a beta-strand and a loop (Fig. 4d) of the same beta-sheet. Sectm1 Ig-like domain may thus form an atypical disulfide bridge while maintaining the Ig-fold. Further structural studies would however be needed to obtain a high resolution 3D structure of Sectm1.
Tex19 and Sectm1 expression patterns are opposite in testis and placenta across species

Both gene expression profiles were investigated across species using publicly available RNA-seq data. In human, Sectm 1 is expressed in a large variety of unrelated tissues, e.g., colon, placenta, kidney, stomach, lung and thymus (Additional file 3). In mouse, it has been established that

Table 5 Template selection for homology modelling of Sectm1 lg-like domain

\begin{tabular}{|c|c|c|c|}
\hline \multicolumn{4}{|c|}{ Sequence similarity between Sectm1 and 3SOB_L antibody light chain (structure available) } \\
\hline & HHpred & high & coring segment between human Sectm 1 and PDB entry $3 S O B \_L$ \\
\hline $\begin{array}{l}\text { Probab }=97.59 \\
E \text {-value }=0.0013 \\
\text { Score }=48.5\end{array}$ & $\begin{array}{l}\text { Sectm1 } \\
3 \mathrm{SOB} \_L \\
\text { Sectm1 } \\
\text { 3SOB_L }\end{array}$ & $\begin{array}{r}14 \\
3 \\
70 \\
63 \\
127 \\
123\end{array}$ & 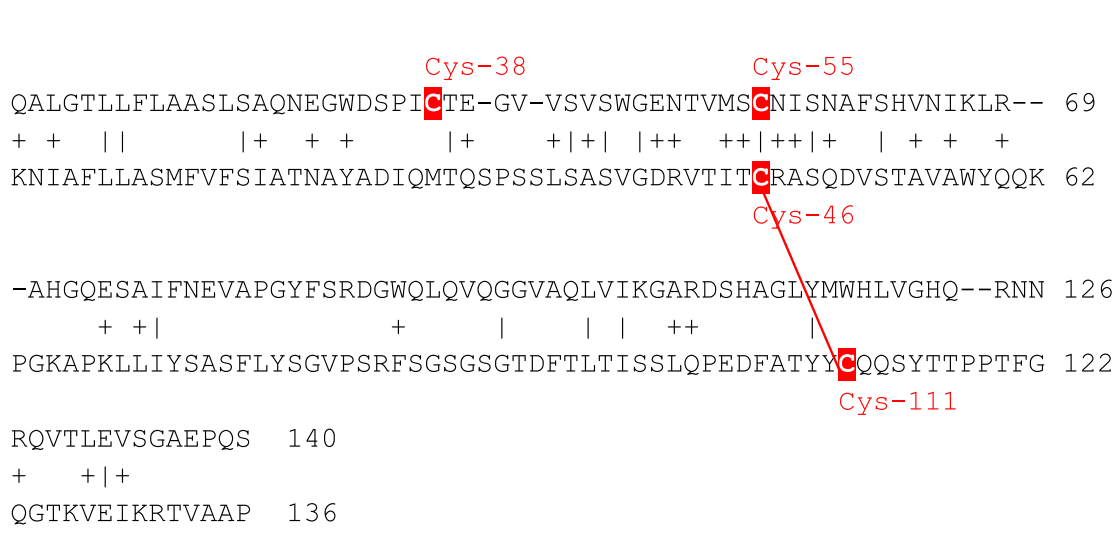 \\
\hline
\end{tabular}


Tex19.1 and Tex19.2 are specifically expressed in reproduction and embryonic tissues, i.e., testis, placenta, ovary and primordial germ cells (PGC) [11]. Therefore, we selected testis and placenta as reference tissues to simultaneously investigate both gene expressions in mouse, cow (or bull) and primates including human. 68 RNA-seq datasets, i.e., 2.8 billion reads, were processed. Liver and heart served as Tex19 negative controls while colon was used as Sectm1 positive control. Moreover, Jurkat-T cells provided a human Tex19 positive control (data not shown). In cow, liver and heart were not strictly negative Sectm1 controls because both tissues expressed Sectm1. Because human, mouse, rat and cow are placental mammals, one may expect some similarity between orthologous gene expression profiles. Surprisingly, we observed heterogeneous or quite opposite patterns of Tex19 and Sectm1 expressions in testis and placenta across species. In testis, human, mouse and rat predominantly express Tex19 while bull mainly expresses Sectm1 (Fig. 5a).
Strikingly, Tex19 and Sectm1 expression levels anticorrelate ( -0.72 Pearson coefficient) across the testis of 5 selected primates (Fig. 5b) which supports anti-regulated gene expressions. In placenta, human expresses Sectm1 but not Tex19 while it is exactly the opposite in mouse (Fig. 5c). For cow, placenta RNA-seq experiments were not available. Therefore, expressed sequence tags (ESTs) were analyzed. EST detect only abundantly expressed genes. Both Sectm1.alpha (7 ESTs) and Sectm1.beta (42 ESTs) transcripts were retrieved by sequence similarity searches (Table 6) (Additional files 4 and 5) and supported Sectm1 abundant expression in cow placenta. Moreover, RT-PCR detected Tex19.alpha but not Tex19.beta expression (Fig. 6a) which means i) that Tex19.alpha is expressed in cow placenta but it is not abundant and ii) Tex19.beta is not expressed at all in cow placenta. In rat, placenta RNA-seq and EST data were unavailable. Using RT-PCR, Tex19.1 was detected but not Tex19.2 expression (Fig. 6b). Therefore, mouse

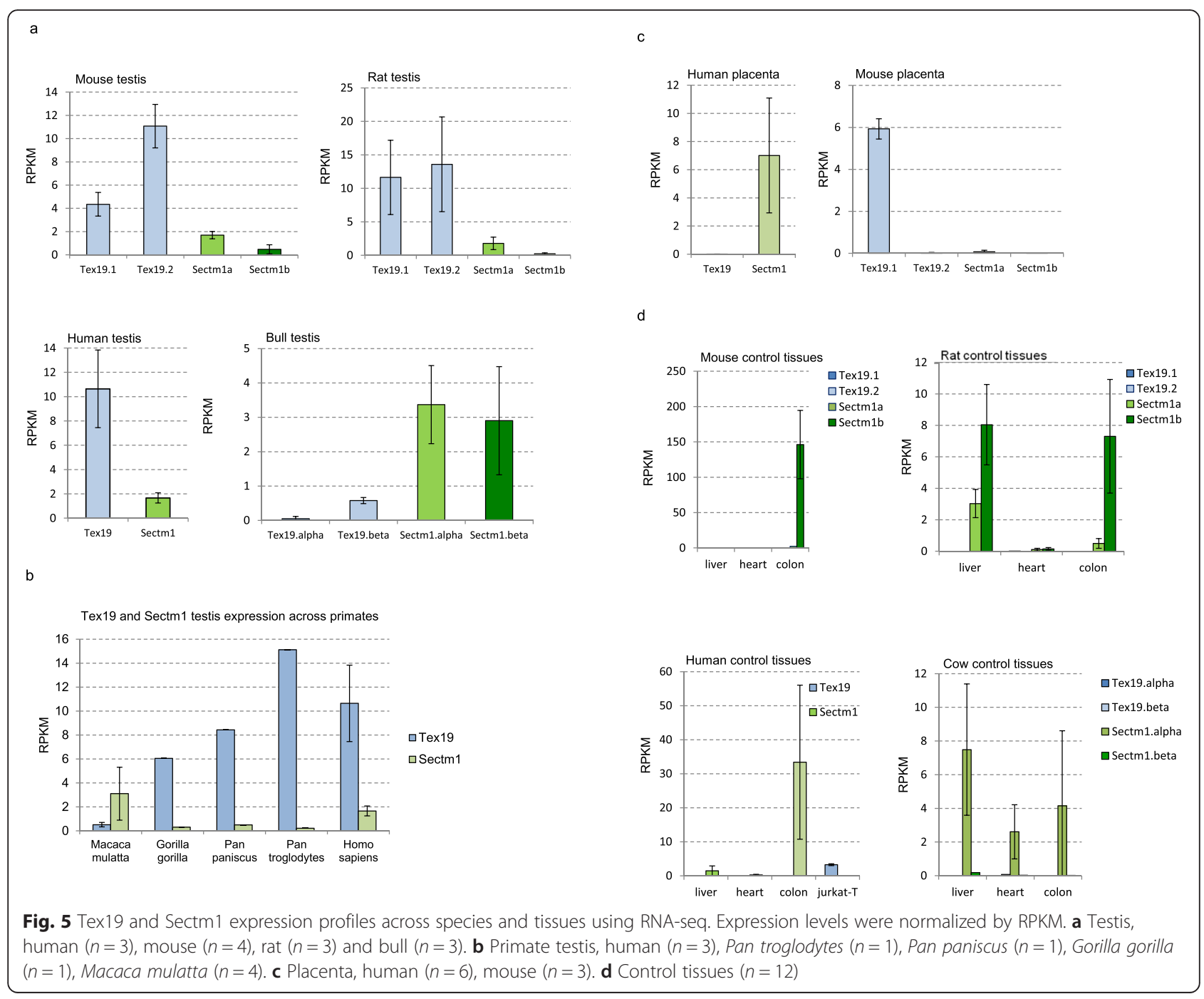


Table 6 Expression profile of Tex19 and Sectm1 transcripts in cow placenta using ESTs

\begin{tabular}{|c|c|c|c|c|c|c|c|c|}
\hline \multirow{2}{*}{$\begin{array}{l}\text { Placenta } \\
\text { EST }\end{array}$} & \multicolumn{2}{|c|}{ Tex19.alpha } & \multicolumn{2}{|c|}{ Tex19.beta } & \multicolumn{2}{|c|}{ Sectm1.alpha } & \multicolumn{2}{|c|}{ Sectm1.beta } \\
\hline & Count & gb_ID & Count & gb_ID & Count & gb_ID & Count & gb_ID \\
\hline \multirow[t]{7}{*}{21,075} & 0 & $\varnothing$ & 0 & $\varnothing$ & 7 & ck394172 & 42 & Add. file 5 \\
\hline & & & & & & aw462349 & & \\
\hline & & & & & & bp110064 & & \\
\hline & & & & & & au233908 & & \\
\hline & & & & & & au278309 & & \\
\hline & & & & & & bp108136 & & \\
\hline & & & & & & au233844 & & \\
\hline
\end{tabular}

gb_ID: genbank record identifiers $\varnothing$ : empty

and rat exhibit similar expression patterns of Tex19 genes in placenta.

\section{Discussion}

Using both molecular phylogeny and statistical test on pattern of gene copy numbers per species, we have demonstrated the co-evolution of Tex19 and Sectm1 genes. Moreover, we have shown that both gene expression patterns are characterized by opposite profiles in reproductive tissues across species. In particular, Tex19 and Sectm1 expression levels anti-correlate in primate testis which brings support to an anti-regulated gene expression. Co-evolution and anti-regulated gene expression strongly suggest a functional relationship between both genes. To determine if the cluster of co-evolving genes extends to CD7 and Uts2r, i.e., Tex19 and Sectm1 neighbors on human and mouse genomes, CD7 and Uts2r accurate molecular phylogenies are required. CD7 and Uts2r phylogenies have not been reported yet. First, a CD7 protein [RefSeq:XP_007482862] and a Uts2r-like protein [RefSeq:XP_007498500] have been predicted in Monodelphis domestica (opossum) which is a marsupial. Second, CD7 has not duplicated in the vicinity of Sectm1 on mouse and rat genomes. Third, Uts2r has not duplicated in the vicinity of Tex19 in the same species. At first glance, CD7 and Uts2r are thus unlikely eutherian-specific and may not co-evolve with Tex19 and Sectm1. At the protein sequence level, we report the presence of 4 invariant cysteines in Tex19. In secreted proteins, invariant cysteines are frequently the signature

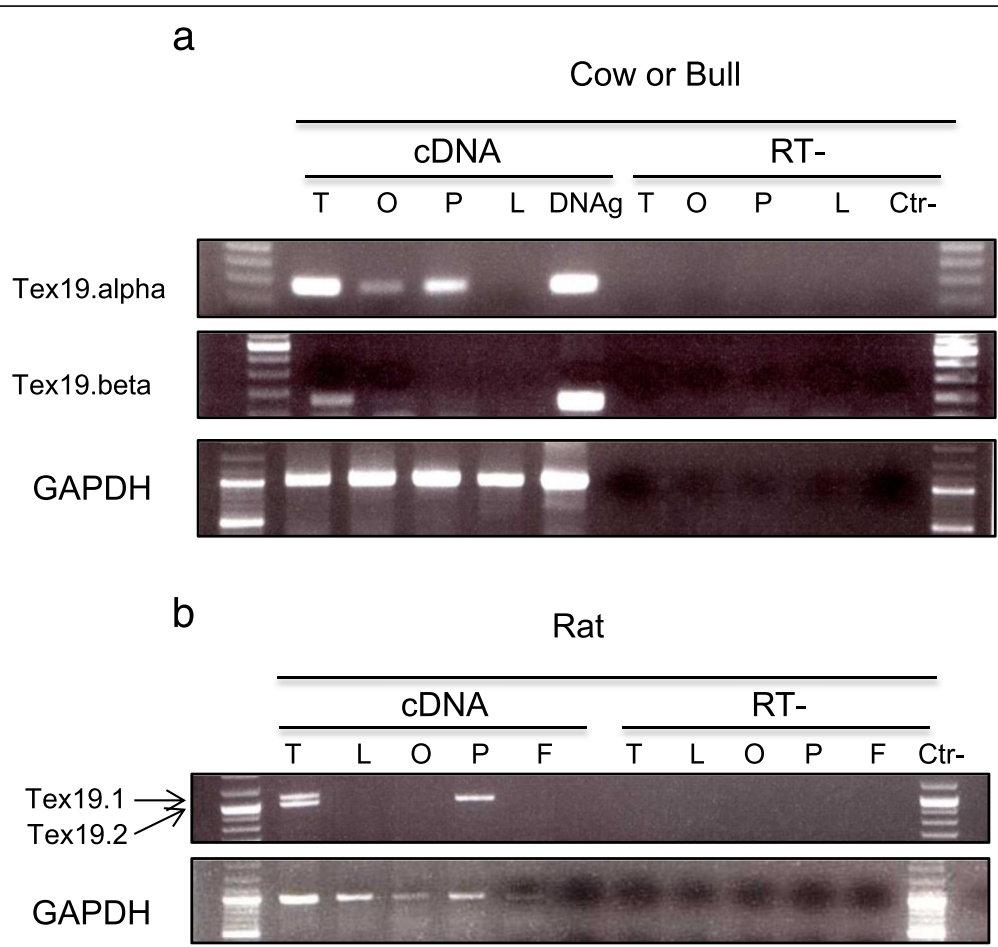

Fig. 6 Reverse Transcription-PCR (RT-PCR). T: testis, O: ovary, P: placenta, L: liver, DNAg: genomic DNA, Ctr-: negative control, RT-: negative retrotranscribed samples. a Tex19.alpha and Tex19.beta RT-PCR in cow. b Tex19.1 and Tex19.2 RT-PCR in rat 
of disulfide bridges that stabilize protein structure [48]. Immunohistochemistry experiments have established that mouse Tex19.1 protein localizes in the cytoplasm $[4,6]$. Cytoplasm is a reductive environment which prevents cysteines from oxidizing into disulfide bridges [48]. Tex19 invariant cysteines are thus intriguing since cytoplasm is not expected to favor disulfide bond formation. In addition, 2 invariant cysteines of the "MCP" region are involved in a $\mathrm{C}_{34} \mathrm{~F}[\mathrm{AT}] \mathrm{C}_{37}[\mathrm{FY}]$ motif which fits the fuzzier CXXC residue pattern. CXXC motifs have been reported in both redox-active catalytic enzymes, e.g., thioredoxin, and zinc fingers $[49,50]$. The high conservation of the "MCP" region and PSIPRED alpha-helices predictions suggest that the region might fold into a stable structural domain. Using homology modelling, we have proposed a 3D model for the Sectm1 Ig-like domain. In the model, the beta-sheets that form the classical Ig sandwich are not linked by a disulfide bridge. Instead, the disulfide is atypically predicted in the same beta-sheet. A similar structure has been previously reported in the cell surface glycoprotein CD4 which is the receptor for human immunodeficiency virus (HIV) [51]. The reason behind Tex19 and Sectm1 co-evolution is a burning question. Co-evolving genes are usually involved in the same biological process, e.g., hormones and receptors. At first glance, Tex19 and Sectm1 act in distinct pathways. On one hand, expression of MMERVK10C endogenous retrovirus is up-regulated during meiosis in the mouse Tex19.1 -/- testis $[4,6]$. In addition, L1 retrotransposon mRNAs are derepressed in mouse Tex19.1 -/- placentas [5]. Therefore, consistent evidences suggest that Tex19 operates a control on TE activity. Expression of mouse Tex19 paralogs has been specifically detected in reproductive tissues, i.e., testis, placenta, ovary and PGC [11]. Moreover, Tex19.1 deletion is associated with spermatogenesis defects and reduction of perinatal survival [6]. Tex19 is thus also involved in species reproduction. On the other hand, Sectm1 is related to the immune system. First, Sectm1 binds $\mathrm{CD} 7$, a $40 \mathrm{kDa}$ protein of the Ig superfamily expressed by natural killer (NK) and T-cells [14]. Second, tumor cells that express Sectm1 attract monocytes [52]. Third, we proposed an Ig-like model for Sectm1 most conserved region by homology to the B cell antigen receptor. The reason why Tex19 and Sectm1 have co-evolved must thus be searched at the interface between TE activity control, species reproduction and immunity. One attractive hypothesis is that Sectm 1 might trigger an immune response against cells that show TE activity while Tex19 could block the immune attack. A mechanism of intrinsic immunity against retrotransposons was previously reported [53]. Moreover, expression of endogenous retrotransposons has been associated with tissue-specific autoimmune diseases [54]. Interestingly, testis and placenta are already known to be immuno-privileged tissues
$[55,56]$, i.e., mechanisms prevent the immune system from attacking male germ cells and embryo which are both antigenic. As a perspective, immunohistochemistry experiments carried out in wild-type and Tex19 knockout mice may prove useful to determine if the behaviour of immune system cells is altered. We are particularly interested to determine if an immune response is raised against testis and placental tissues that do not express any more Tex19 genes. Conversely, a knock-out of Sectm1 might be associated with uncontrolled expression of TE and Tex19. In our study, we showed that Tex19 and Sectm1 expression levels anti-correlate in the testis of several primates which supports anti-regulated gene expressions. Tex19 expression profile was compared in the testis of Homo sapiens, Pan troglodytes, Pan paniscus, Gorilla gorilla and Macaca mulatta. It is interesting to note that human, both chimpanzees and gorilla belong to the Hominidae family while Macaca belongs to the Cercopithecidae family. In testis, Hominidae show greater Tex19 expression than Sectm1 while it is the opposite in Macaca mulatta. It would be interesting to measure the level of expression of both genes in the testis of further Cercopithecidae primates, e.g., Papio anubis and Chlorocebus sabaeus, to determine if greater Sectm1 expression is Cercopithecidae-specific. However, drawing any interpretation on primate physiology based on Tex19 and Sectm1 expression profiles alone would be far-fetched. Although human Tex19 and Sectm1 are transcribed in opposite directions, both genes are separated by a $25 \mathrm{~kb}$ distance which is too long to support a bidirectional promoter. Indeed, bidirectional promoters are known to be short DNA sequences [57], i.e., less than $1 \mathrm{~kb}$. However, it is possible that Tex19 and Sectm1 genes share regulatory elements in their promoter regions and compete for transcription factor binding. Sequence analysis of both promoters is required to test this hypothesis. Moreover, both protein functions may be opposed. Antigenic cells that express Tex19 could escape the immune system. The relation between Tex19 and Sectm1 has been also striking at the protein organization level. Haplorrhini primates lost Tex19 C-terminal region ("iLeu" conserved region) while a Sectm1 extra C-terminal domain was acquired (conserved region B). However, Tex19 C-terminal region "iLeu" and Sectm1 extra C-terminal domain do not share any sequence similarity. Of note, human Sectm1 is expressed in a large variety of tissues, e.g., colon, placenta, leukocytes, kidney and stomach and we were able to identify many tissues that express Sectm1 but not Tex19, e.g., colon. Therefore, Sectm 1 function does not necessarily require the co-presence of Tex19. Cells that express Sectm1 but not Tex19 may not be capable of immuno-suppression. Interestingly, we also detected Tex19 expression in human neuroblastoma, rhabdomyosarcoma and Jurkat T-cells, i.e., a leukemia cell line. It is not known if those cells show TE 
activity and if there is a benefit for cancer cells to express Tex19. We have also demonstrated the eutherian specificity of Tex19 and Sectm1. The gene system appeared 100 million years ago in the eutherian common ancestor [58] and was transmitted to every placental mammals. We have not retrieved any species that exhibit a Sectm1 but not a Tex19 gene or the opposite except Dasypus novemcinctus (Xenarthra) whose genome is not complete yet. Therefore, Tex19 and Sectm1 gene system is likely to play a critical role for eutherian biology. We noticed that expression patterns of Tex19 and Sectm1 are heterogeneous across tissues and species. A striking example is placenta. Using RNA-seq data, we observed that human placenta expresses Sectm1 but not Tex19 while it is the opposite in mouse. Differences in Tex19 and Sectm1 expression may be associated with specific tuning of TE activity control across tissues and species. However, it is currently not known if TE repression and Tex19 expression level correlate.

\section{Conclusions}

Using molecular phylogeny and statistical tests on pattern of gene copies across species, we have established the co-evolution and eutherian specificity of Tex19 and Sectm1 genes. Tex19 and Sectm1 are involved in distinct biological processes, i.e., retrotransposon activity control and immunity, respectively. Sectm1 shows a broad range of tissue expression while Tex19 is specifically expressed in reproductive tissues and PGC. In addition, Tex19 and Sectm1 expression levels anti-correlate in primate testis. Our results suggest a strong functional relationship between both genes. Tex19 might suppress an immune response triggered by Sectm1 against cells that show TE activity. If the function relation between both genes could be clearly identified, new light could be shed on eutherian evolution, pathologies and biological processes as diverse as male sterility, pregnancy miscarriage, immune system evasion of tumors and auto-immune diseases.

\section{Additional files}

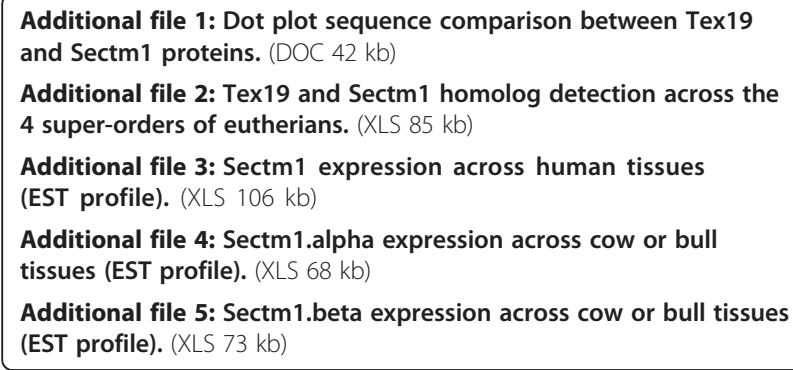

\section{Abbreviations}

Tex19: Testis expressed 19; Sectm1: Secreted and transmembrane 1; ML: Maximum likelihood; TE: Transposable elements; MACS: Multiple alignment of complete protein sequences; Ig: Immunoglobulin; SP: Secretion peptide; LINE: Long interspersed nuclear element; SINE: Short interspersed nuclear element; LTR: Long terminal repeat; ERV: Endogenous retroviruses; CDS: Coding sequence; TM: Transmembrane; KO: Knock-out; HTP: Highthroughput; SRA: Sequence read archive; PDB: Protein Data Bank; WGS: Whole genome shotgun; PSI-BLAST: Position specific iterated BLAST; NCBI: National center for biotechnology information; IRE: Interspersed repetitive elements; EST: Expressed sequence tag; RT-PCR: Reverse-transcription polymerase chain reaction; Uts2r: Urotensin 2 receptor; PGC: Primordial germ cells.

\section{Competing interests}

The authors have no competing interest to declare.

\section{Authors' contributions}

LB- project leader, bioinformatics analysis and manuscript preparation, YT- RNA extraction, RT-PCRs and Tex19 specialist, OL- bioinformatics expertise and manuscript correction, RS- molecular model validation, OP- comparative genomics expertise and manuscript correction, AD- molecular modelling expertise, project follow-up and manuscript correction, SV- Tex19 specialist, project initiation, follow-up and manuscript correction. All authors read and approved the final manuscript.

\section{Acknowledgements}

This work was supported by the National Institute of Health and Medical Research (INSERM), the National Center of Scientific Research (CNRS) and the Strasbourg University (UdS). We are grateful to Dr Marius Télétin for helpful comments. We express our gratitude to Prof. Catherine Eltchebest. We thank Dr. Corinne Di Trani and Dr. David Di Paolo (Zoological and botanical park of Mulhouse, France) for stimulating discussions. Finally, Laurent Bianchetti would like to thank Dr. Gula Yusupova, Dr. Patrick Schultz, Dr. Christelle Thibault-Carpentier, Dr Isabelle Lebars and Dr. Stéphanie Kleinlogel for their support.

\section{Author details}

${ }^{1}$ Biocomputing and Molecular Modelling Laboratory, Integrated Structural Biology Department, Genetics institute of Molecular and Cellular Biology (IGBMC), INSERM U964/CNRS UMR 1704/Strasbourg University, 1 rue Laurent Fries, 67404 Illkirch, France. ${ }^{2}$ Primordial Germ Cells' Ontogeny and

Pluripotency Laboratory, Functional Genomics and Cancer Department, Genetics Institute of Molecular and Cellular Biology (IGBMC), INSERM U964/ CNRS UMR 1704/Université de Strasbourg, 1 rue Laurent Fries, 67404 IIlkirch, France. ${ }^{3}$ Bioinformatics and Integrated Genomics Laboratory (LBGI), ICube, CNRS UMR 7357/Université de Strasbourg, 11 rue Humann, 67085 Strasbourg, France. ${ }^{4}$ Centre Hospitalier Universitaire, 67000 Strasbourg, France. ${ }^{5}$ Present address: Institut de génétique humaine (IGH), 141 rue de la Cardonille, 34396 Montpellier, France.

Received: 2 April 2015 Accepted: 1 October 2015

Published online: 12 October 2015

\section{References}

1. Kumar CS, Qureshi SF, Ali A, Satyanarayana ML, Rangaraju A, Venkateshwari A, et al. Hidden magicians of genome evolution. Indian J Med Res. 2013;137(6):1052-60.

2. Wicker T, Sabot F, Hua-Van A, Bennetzen JL, Capy P, Chalhoub B, et al. A unified classification system for eukaryotic transposable elements. Nat Rev Genet. 2007;8(12):973-82.

3. Zamudio N, Bourc'his D. Transposable elements in the mammalian germline: a comfortable niche or a deadly trap ? Heredity. 2010;105(1):92-104.

4. Ollinger R, Childs AJ, Burgess HM, Speed RM, Lundegaard PR, Reynolds N, et al. Deletion of the pluripotency-associated Tex19.1 gene causes activation of endogenous retroviruses and defective spermatogenesis in mice. Plos Gen. 2008:4:e1000199.

5. Reichmann J, Reddington JP, Best D, Read D, Ollinger R, Meehan RR, et al. The genome-defence gene Tex19.1 suppresses LINE-1 retrotransposons in the placenta and prevents intra-uterine growth retardation in mice. Hum Mol Genet. 2013;22(9):1791-806.

6. Tarabay Y, Kieffer E, Teletin M, Celebi C, van Montfoort A, Zamudio N, et al. The mammalian-specific Tex19.1 gene plays an essential role in spermatogenesis and placenta-supported development. Hum Reprod. 2013;28(8):2201-14.

7. Kuntz S, Kieffer E, Bianchetti L, Lamoureux N, Furhmann G, Viville S. Tex19, a mammalian-specific protein with a restricted expression in pluripotent stem cells and germ line. Stem Cells. 2008;26(3):734-44. 
8. Tautz D, Domazet-Loso T. The evolutionary origin of orphan genes. Nat Rev Genet. 2011;12(10):692-702.

9. Wang PJ, McCarrey JR, Yang F, Page DC. An abundance of X-linked genes expressed in spermatogonia. Nat Genet. 2001;27(4):422-6.

10. Prosdocimi F, Linard B, Pontarotti P, Poch O, Thompson JD. Controversies in modern evolutionary biology: the imperative for error detection and quality control. BMC Genomics. 2012;13:5.

11. Celebi C, van Montfoort A, Skory V, Kieffer E, Kuntz S, Mark M, et al. Tex 19 paralogs exhibit a gonad and placenta-specific expression in the mouse. J Reprod Dev. 2012:58(3):360-5.

12. Slentz-Kesler KA, Hale LP, Kaufman RE. Identification and characterization of K12 (SECTM1), a novel human gene that encodes a Golgi-associated protein with transmembrane and secreted isoforms. Genomics. 1998:47(3):327-40.

13. Lyman SD, Escobar S, Rousseau AM, Armstrong A, Fanslow WC. Identification of CD7 as a cognate of the human K12 (SECTM1) protein. J Biol Chem. 2000;275(5):3431-7.

14. Howie D, Garcia Rueda H, Brown MH, Waldmann H. Secreted and transmembrane $1 \mathrm{~A}$ is a novel co-stimulatory ligand. Plos one. 2013;8(9):e73610.

15. Lam GK, Liao HX, Xue Y, Alam SM, Scearce RM, Kaufman RE, et al. Expression of the CD7 ligand K-12 in human thymic epithelial cells: regulation by IFN-gamma. J Clin Immunol. 2005;25(1):41-9.

16. Liu L, Li Y, Li S, Hu N, He Y, Pong R, et al. Comparison of next-generation sequencing systems. J Biomed Biotechnol. 2012;251364.

17. Henson J, Tischler G, Ning Z. Next generation sequencing and large genome assemblies. Pharmacogenomics. 2012;13(8):901-15.

18. Lindblad-Toh K, Garber M, Zuk O, Lin MF, Parker BJ, Washietl S, et al. A high-resolution map of human evolutionary constraint using 29 mammals. Nature. 2011;478(7370):476-82

19. Asher RJ, Bennett N, Lehmann T. The new framework for understanding placental mammal evolution. Bioessays. 2009;31(8):853-64.

20. English AC, Richards S, Han Y, Wang M, Vee V, Qu J, et al. Mind the gap: upgrading genomes with Pacific Biosciences RS long-read sequencing technology. Plos One. 2012;7(11):e47768.

21. Warren WC et al. Genome analysis of the platypus reveals unique signatures of evolution. Nature. 2008;453(7192):175-83.

22. Renfree MB et al. Genome sequence of an Australian kangaroo, Macropus eugenii, provides insight into the evolution of mammalian reproduction and development. Genome Biol. 2011;12(18):R81.

23. Lecompte O, Thompson JD, Plewniak F, Thierry J, Poch O. Multiple alignment of complete sequences (MACS) in the post-genomic era. Gene. 2001;270(1-2):17-30

24. Ochoa D, Pazos F. Studying the co-evolution of proteins families with the Mirrortree web server. Bioinformatics. 2010;26(10):1370-1.

25. Pazos F, Valencia A. Protein co-evolution, co-adaptation and interactions. EMBO J. 2008:27(20):2648-55.

26. Fryxell KJ. The coevolution of gene family trees. Trends Genet. 1996:12(9):364-9.

27. Van Kesteren RE, Tensen CP, Smit AB, van Minnen J, Kolakowski LF, Meyerhof W, et al. Co-evolution of ligand-receptor pairs in the vasopressin/oxytocin superfamily of bioactive peptides. J Biol Chem. 1996;271(7):3619-26.

28. Kawaguchi M, Inoue K, Luchi I, Nishida M, Yasumasu S. Molecular co-evolution of a protease and its substrate elucidated by analysis of the activity of predicted ancestral hatching enzyme. BMC Evol Biol. 2013;13:231.

29. Jacob F, Monod J. Genetic regulatory mechanisms in the synthesis of proteins. J Mol Biol. 1961:3:318-56.

30. Michalak P. Coexpression, coregulation and cofunctionality of neighboring genes in eukaryotic genomes. Genomics. 2008;91(3):243-8.

31. Thévenin A, Ein-Dor L, Ozery-Flato M, Shamir R. Functional gene groups are concentrated within chromosomes, among chromosomes and in the nuclear space of the human genome. Nucleic Acids Res. 2014;42(15):9854-61.

32. Brayton KA, Zhengdao C, Zhou G, Nagy PL, Gavalas A, Trent JM, et al. Two genes for de novo purine nucleotide synthesis on human chromosome 4 are closely linked and divergently transcribed. J Biol Chem. 1994;269(7):5313-2.

33. Blumenthal T, Evans D, Link CD, Guffanti A, Lawson D, Thierry-Mieg J, et al. A global analysis of Caenorhabditis elegans operons. Nature. 2002:417:851-4.

34. Brawand D, Soumillon M, Necsulea A, Julien P, Csardi G, Harrigan P, et al. The evolution of gene expression levels in mammalian organs. Nature. 2011;478(7369):343-8.
35. Merkin J, Russell C, Chen P, Burge CB. Evolutionary dynamics of gene and isoform regulation in Mammalian tissues. Science. 2012;338(6114):1593-9.

36. Türeci O, Koslowski M, Helftenbein G, Castle J, Rohde C, Dhaene K, et al. Claudin-18 gene structure, regulation, and expression is evolutionary conserved in mammals. Gene. 2011;481(2):83-92.

37. Cridland JA, Curley EZ, Wykes MN, Schroder K, Sweet MJ, Roberts TL, et al. The mammalian PYHIN gene family: phylogeny, evolution and expression. BMC Evol Biol. 2012;12:140.

38. Kodama $Y$, Shumway $M$, Leinonen $R$, International Nucleotide Sequence Database Collaboration. The Sequence Read Archive: explosive growth of sequencing data. Nucleic Acids Res. 2012;40(Database issue):D54-6.

39. Plewniak F, Bianchetti L, Brelivet $Y$, Carles A, Chalmel F, Lecompte O, et al. PipeAlign: a new toolkit for protein family analysis. Nucleic Acids Res. 2003;31(13):3929-832.

40. Waterhouse AM, Procter JB, Martin DM, Clamp M, Barton GJ. Jalview Version 2 - a multiple sequence alignment editor and analysis workbench. Bioinformatics. 2009;25(9):1189-91.

41. Crooks GE, Hon G, Chandonia JM, Brenner SE. WebLogo: a sequence logo generator. Genome Res. 2004;14(6):1188-90.

42. Tamura K, Peterson D, Peterson N, Stecher G, Nei M, Kumar S. MEGA5: molecular evolutionary genetics analysis using maximum likelihood, evolutionary distance, and maximum parsimony methods. Mol Biol Evol. 2011;28(10):2731-9.

43. Abascal F, Zardoya R, Posada D. ProtTest: selection of best-fit models of protein evolution. Bioinformatics. 2005;21(9):2104-5.

44. Letunic I, Bork P. Interactive Tree Of Life v2: online annotation and display of phylogenetic trees made easy. Nucleic Acids Res. 2011;39(Web Server issue):W475-478.

45. Fariselli P, Rossi I, Capriotti E, Casadio R. The WWWH of remote homolog detection: the state of the art. Brief Bioinform. 2007;8(2):78-87.

46. Söding J, Biegert A, Lupas AN. The HHpred interactive server for protein homology detection and structure prediction. Nucleic Acids Res. 2005;33(Web server issue):W244-248.

47. Branden C, Tooze J. From Recognition of Foreign Molecules by the Immune System. In: Garland publishing group, editor. Introduction to protein structure. 2nd ed. 1998. p. 299-323.

48. Wiederstein M, Sippl MJ. ProSA-web: interactive web service for the recognition of errors in three-dimensional structures of proteins. Nucleic Acids Res. 2007;35(Web server issue):W407-10.

49. Bosnjak I, Bojovic V, Segvic-Bubic T, Bielen A. Occurrence of protein disulfide bonds in different domains of life: a comparison of proteins from the Protein Data Bank. Protein Eng Des Sel. 2014:27(3):65-72.

50. Krishna SS, Majumdar I, Grishin NV. Structural classification of zinc fingers: survey and summary. Nucleic Acids Res. 2003;31(2):532-50.

51. Wang J, Yan Y, Garrett TPJ, Liu J, Rodgers DW, Garlick RL, et al. Atomic structure of a fragment of human CD4 containing two immunoglobulin-like domains. Nature. 1990;348:411-8.

52. Wang T, Ge Y, Xiao M, Lopez-Coral A, Li L, Roesch A, et al. SECTM1 produced by tumor cells attracts human monocytes via CD7-mediated activation of the PI3K pathway. J Invest Dermatol. 2014;134(4):1108-18.

53. Koito A, Ikeda T. Intrinsic immunity against retrotransposons by APOBEC cytidine deaminases. Front Microbiol. 2013;4:28.

54. Volkman HE, Stetson DB. The enemy within: endogenous retroelements and autoimmune disease. Nat Immunol. 2014;15(5):415-22.

55. Moffett A, Loke C. Immunology of placentation in eutherian mammals. Nat Rev Immunol. 2006;6(8):584-94.

56. Fijak M, Meinhardt A. The testis in immune privilege. Immunol Rev. 2006;213:66-81.

57. Trinklein ND, Aldred SF, Hartman SJ, Schroeder DI, Otillar RP, Myers RM. An abundance of bidirectional promoters in the human genome. Genome Res. 2004:14:62-6.

58. Genome 10K Community of Scientists. Genome 10K: a proposal to obtain whole-genome sequence for 10,000 vertebrate species. J Heredity. 2009:100(6):659-74. 